\title{
Ações biológicas de compostos de selênio e telúrio: efeitos tóxicos sobre o sistema nervoso central
}

Flavia Carla Meotti

Cristina Wayne Nogueira

\section{Resumo}

A bioquímica e a farmacologia do selênio têm sido assunto de muito interesse, particularmente sob o ponto de vista de saúde pública. O selênio há muito tempo descrito como um antioxidante, e agora também conhecido por ser um componente essencial do sítio ativo de diversas enzimas. Ao contrário do selênio, pouco é conhecido sobre a ocorrência do telúrio em sistemas biológicos. Apesar de não apresentar função fisiológica descrita, o telúrio compartilha algumas peculiaridades químicas com o selênio. Neste contexto, pesquisas que revelem as ações biológicas de moléculas que contenham átomos de selênio e telúrio mostram-se de grande importância em virtude de suas aplicações como ferramentas sínteticas em química orgânica e de suas propriedades farmacológicas. Paralelo a isso, já se sabe que moléculas que contenham selênio e telúrio são capazes de reagir com grupos $\mathrm{SH}$ de proteínas biologicamente ativas. Com base nesta característica, estudos revelam que estes compostos são potencialmente tóxicos ao sistema nervoso central (SNC). Sendo assim, esta revisão aborda os efeitos tóxicos induzidos por compostos orgânicos de selênio e telúrio, com ênfase nos efeitos sobre o SNC. 


\section{Abstract}

The biochemistry and pharmacology of selenium is a subject of intense current interest, particularly from the viewpoint of public health. Selenium, long recognized as a dietary oxidant, is now known to be an essential component of the active sites of several enzymes. In contrast to the case of selenium, surprisingly little is known of the occurrence of tellurium in biological systems. However, tellurium shares chemical characteristics with selenium. Taking into account, researches that focus on biological activities of selenium and tellurium are the greatest interest. These compounds are important intermediates in organic synthesis and present some pharmacological properties. Besides, selenium and tellurium can oxidize $\mathrm{SH}$ groups of proteins since they could be toxic to animals. Based in this property, some studies revealed that selenium and tellurium are potentially neurotoxic. In this way, this review point out toxic effects induced by selenium and tellurium organic compounds highlight the effects on central nervous system.

\section{Selênio}

\subsection{Histórico}

Os primeiros relatos da intoxicação por selênio foram descritos por Marco Polo no século XIII, quando cavalos na região de Succuir, no oeste da China apresentavam perda de cascos e pêlos após a ingestão de plantas venenosas. Seis séculos após os relatos de Marco Polo, foram evidenciados os mesmos sintomas em animais que se alimentavam da vegetação nativa próxima ao Rio Missouri, entre o Sul de Dakota e Nebraska. A descoberta do agente etiológico destes efeitos tóxicos data do ano de 1928, quando o Doutor Kurt Franke estudou estas plantas e seus grãos, concluindo que se tratava de selênio (FRANKE, 1934). Em 1933, foi feita pela primeira vez uma associação entre os relatos de Marco Polo e uma doença denominada "alkali disease" que acometia animais de fazenda, nos EUA (SPALLHOLZ, 1993).

Devido a estas intoxicações, o selênio passou a ser um elemento conhecido por sua importância toxicológica, pois causava emagrecimento, perda de pêlos e anemia (FRANKE, 1934). Investigando-se a respeito das fontes naturais de selênio, foi descoberto que algumas espécies de plantas dos gêneros Astragalus, Xylorrhiza, Oonopsis e Stanleya eram capazes de acumular este elemento quando cresciam em solos seleníferos (TRELEASE \& BEATH, 1949). 
Estas espécies de plantas provavelmente estão relacionadas com os relatos de Marco Polo no século XIII.

Por outro lado, o selênio é um elemento traço, cuja essencialidade nutricional foi demonstrada em 1957 em ratos (SCHWARTZ \& FOLTZ, 1957). Nos últimos anos, tem sido descrito que baixos níveis de selênio podem levar à predisposição para o desenvolvimento de algumas doenças, tais como câncer, esclerose cardiovascular, cirrose e diabetes (NAVARRO-ALARCÓN \& LÓPEZMARTÍNEZ, 2000). A deficiência de selênio em humanos também pode causar: problemas musculares, alterações digestivas, doenças cardiovasculares e alterações reumáticas (NEVE et al., 1987; ORTUÑO et al., 1996).

O selênio é um micronutriente cuja concentração pode ocasionar deficiência ou toxicidade, portanto é de grande interesse determinar a influência da suplementação de diferentes formas de selênio e sua biodisponibilidade. Estudos com animais demonstraram que a biodisponibilidade das formas orgânicas de selênio (Semetionina) são maiores do que as obtidas nas formas inorgânicas (selenito e selenato) (LEVANDER et al., 1983; SMITH \& PICCIANO, 1987). Observações semelhantes foram obtidas em humanos (LITOV \& COMBS, 1991; FAVIER, 1993).

Neste contexto, a suplementação de dietas com selênio, tanto para animais quanto para humanos, tem sido aceita pela comunidade científica. A Junta de Alimentação e Nutrição da Academia de Ciências dos Estados Unidos propõe uma ingestão diária de 50-200 mg/dia, a qual é considerada segura e saudável para adultos (FOOD AND NUTRITION BOARD, 1989).

\subsection{Características Químicas}

O selênio é um elemento do grupo 16 da tabela periódica, podendo apresentar-se sob quatro estados de oxidação: selenato $(\mathrm{Se}+6)$, selenito $(\mathrm{Se}+4)$, selênio elementar $(\mathrm{Se})$ e seleneto $(\mathrm{Se}-2)$.

A similaridade nas propriedades químicas do enxofre e do selênio tem estimulado a síntese e o estudo, com fins comparativos, de uma grande variedade de selenomoléculas derivadas de compostos que contenham enxofre (PARNHAM \& GRAF, 1991); entretanto, as diferenças nas propriedades físico-químicas entre selênio e enxofre constituem as bases de seus papéis biológicos específicos (STADTMAN, 1980). Os selenóis (R-SeH) são as formas correspondentes aos tióis (R-SH), onde ocorre a substituição do átomo de 
enxofre pelo átomo de selênio (KLAYMAN \& GÜNTHER, 1973). Este elemento diminui a toxicidade de metais pesados, tais como o mercúrio, o chumbo e a prata (FROST, 1983; CUVIN-ARALAR \& FURNESS, 1991; ELLINGSEN et al., 1993; LEVANDER \& BURK, 1994).

\subsection{Atividades Biológicas}

O selênio apresenta um grande número de funções biológicas, sendo mais importante a de antioxidante.

As espécies reativas de oxigênio (ERO) são produzidas normalmente durante o metabolismo celular. As ERO incluem radicais hidroxil $\left(\mathrm{OH}^{\circ}\right)$ e ânion superóxido $\left(\mathrm{O}_{2}{ }^{\circ}\right)$, bem como peróxido de hidrogênio $\left(\mathrm{H}_{2} \mathrm{O}_{2}\right)$ entre outros. Os radicais livres possuem uma grande reatividade e podem levar à lipoperoxidação, oxidação de carboidratos, proteínas e DNA (PRATICÒ \& DELANTY, 2000). As ERO podem ser tóxicas e determinantes do tempo de vida celular (PARKES et al., 1998).

Estudos recentes demonstram que os metabólitos das ERO estão associados com muitos processos degenerativos (MELOV et al., 1998; JANSSEN et al., 1998). Alterações no status oxidativo do organismo têm sido implicadas em diversas desordens, tais como câncer, catarata, isquemia, enfisema pulmonar, diabetes mellitus, envelhecimento, doenças neurodegenerativas e cirrose hepática (COHEN, 1989; HALLIWELL \& GUTTERIDGE, 1990; FLOYD, 1990). As ERO estão aumentadas durante o episódio convulsivo e tanto a iniciação quanto a propagação da lipoperoxidação têm um papel na epileptogênese (WILLMORE, 1990).

Os antioxidantes são substâncias que direta ou indiretamente protegem os sistemas celulares dos efeitos tóxicos produzidos por radicais oxidativos (HALLIWELL, 1995). Existem diversos compostos com ação biológica e importante função antioxidante, entre eles: vitamina C, glutationa, glutationa peroxidase (GSH-Px), superóxido dismutase e catalase (KRISHNA et al., 1996; EVANS et al., 1997; JOURD'HEUIL et al., 1998; McKENZIE et al., 1998; HALLIWELL, 1999 ).

O selênio tem importância fisiológica por participar do sítio ativo de algumas enzimas, dentre elas a glutationa peroxidase (GSHPx). Esta enzima catalisa a redução de hidroperóxidos utilizando-se de grupos tiólicos (glutationa) como doador de elétrons (agente redutor), protegendo o organismo dos danos oxidativos (MATÉS, 2000). 
O selênio também faz parte de outras selenoproteínas funcionalmente ativas, tais como: 5'-deiodinase (BEHNÉ \& KYRIAKOPOULOS, 1990) e fosfolipídio hidroperóxido glutationa peroxidase (URSINI et al., 1982).

Além do importante papel nas reações de redução de hidroperóxidos, a glutationa peroxidase participa da via da lipooxigenase, em sistemas antioxidantes orgânicos junto com a catalase, superóxido dismutase, vitaminas $\mathrm{E}$ e $\mathrm{C}$ e carotenóides, cuja principal função é eliminar as ERO, como peróxido de hidrogênio, hidroperóxidos orgânicos, superóxidos e radicais hidroxil. A eficiente remoção destes radicais livres mantém a integridade das membranas, reduz o risco de câncer, reduz o processo de envelhecimento e doenças degenerativas (NAVARRO-ALARCÓN \& LÓPEZMARTÍNEZ, 2000).

A seqüência de reações proposta para a enzima glutationa peroxidase ocorre em três etapas (Esquema 1). Inicialmente a selenocisteína presente no sítio ativo da enzima reage com um hidroperóxido orgânico para formar ácido selenínico, a seguir ocorre uma redução seqüencial (duas etapas) da enzima com oxidação da glutationa (GSH) (SIES, 1993).

\section{E-Cys-SeH + ROOH $\rightarrow$ E-Cys-SeOH + ROH \\ $\mathrm{E}-\mathrm{Cys}-\mathrm{SeOH}+\mathrm{GSH} \rightarrow \mathrm{E}-\mathrm{Cys}-\mathrm{Se}-\mathrm{SG}+\mathrm{H} 2 \mathrm{O}$ \\ E-Cys-Se-SG + GSH $\rightarrow$ E-Cys-SeH + GSSG}

Esquema 1. Redução de hidroperóxidos pela GSH-Px (SIES, 1993)

Com a descoberta do papel essencial do selênio no centro ativo da enzima glutationa peroxidase (ROTRUCK et al., 1981) e com o aumento do entendimento do papel fisiológico do selênio na regulação do dano oxidativo (CADENAS \& SIES, 1985; URSINI \& BINDOLI, 1987), aumentou o interesse na síntese de compostos orgânicos que contenham selênio e que possuam propriedades biológicas e aplicações farmacológicas (PARNHAM \& GRAF, 1991).

O composto orgânico de selênio, Ebselen (2-fenil-1,2- 
benzisoselenazol-3(2H)-one), apresenta atividade tiol peroxidase intrínseca (MÜLLER et al., 1984; PARNHAM \& GRAF, 1991; DAIBER et al., 2000), semelhante a da selenoenzima glutationa peroxidase. Seu efeito terapêutico está, provavelmente, associado a esta atividade catalítica (MÜLLER et al., 1984; WENDEL et al., 1984).

O Ebselen tem sido usado como antioxidante, possui atividade neuroprotetora em cultura de neurônios (TAN et al., 1997), inibe o dano oxidativo ao DNA, induzido pela dopamina, na presença de íons cobre (LI \& CAO, 2002), protege contra a excitotoxicidade induzida pelo glutamato (PORCÍ́NCULA et al., 2001), reduz o dano oxidativo, em cérebro, causado pela isquemia (IMAl et al., 2003). Além de possuir atividade antiinflamatória (PARNHAM \& GRAF, 1991) e antinociceptiva (NOGUEIRA et al., 2003b).

Em modelos com primatas, o Ebselen reduz os sintomas da doença de Parkinson induzidos pelo ion 1-metil - 4- fenilpiridina (MPP+) (MOUSSAOUI et al., 2000). Devido aos diversos indicativos clínicos de redução de danos cerebrais após aneurisma, este composto consiste em um agente neuroprotetor promissor (SAITO et al., 1998). A injeção intraestriatal de Ebselen protege contra a peroxidação lipídica induzida por ácido quinolínico em ratos (ROSSATO et al., 2002b). Corroborando com estas evidências, o Ebselen vem sendo utilizado no tratamento clínico de pacientes com isquemia aguda (YAMAGUCHI et al., 1998; KONDOH et al., 1999).

O mecanismo exato pelo qual o Ebselen exerce atividade neuroprotetora ainda não está completamente estabelecido, mas está, certamente, relacionado a suas propriedades antioxidantes e antiinflamatórias (TAKASAGO et. al., 1997; SAITO et al., 1998)

Outros compostos orgânicos de selênio, tais como disseleneto de difenila, também possuem atividade tiol-peroxidase e propriedades antioxidantes (WILSON et al., 1989; ROSSATO et al., 2002a). Disseleneto de difenila possui atividade antiinflamatória e antinociceptiva para ratos e camundongos em doses não tóxicas (NOGUEIRA et al., 2003b).

\subsection{Toxicologia}

Durante os últimos anos tem crescido o interesse em relação ao selênio, devido ao seu papel tanto como elemento tóxico quanto essencial (NAVARRO-ALARCÓN \& LÓPEZ-MARTÍNEZ, 2000).

O homem é suscetível à intoxicação por selênio, principalmente devido ao seu amplo uso na indústria (WILBER, 1980), parti- 
cularmente na síntese de compostos, uma vez que este elemento constitui um importante intermediário em síntese orgânica (COMASSETO et al., 1997).

Quando a ingestão diária de selênio excede a capacidade corporal de eliminação pode haver intoxicação e os sintomas crônicos mais comuns são: severa irritação das vias respiratórias, gosto tipicamente metálico na boca, edema pulmonar e cheiro característico de "alho" (BEDWALL et al., 1993; DIAZ et al., 1997).

Em animais intoxicados cronicamente, o selênio é depositado, principalmente, nos rins e fígado, seguido pelo pâncreas, baço e pulmões (WILBER, 1980). O principal local de absorção parece ser o duodeno, seguido pelo jejuno e íleo. Além do trato gastrointestinal, o selênio pode ser absorvido por tecidos cutâneos ou por inalação. Estas duas últimas vias de absorção estão relacionadas com a exposição e intoxicação ocupacional por compostos de selênio (WHANGER et al., 1976). A toxicidade induzida por disselenetos orgânicos difere conforme a via de administração e também segundo a espécie animal tratada (NOGUEIRA et al., 2003a; MEOTTI et al., 2003).

Até o presente momento, os mecanismos envolvidos na toxicidade do selênio ainda não estão bem definidos. Entretanto, PAINTER (1941) propôs que a toxicidade do selenito era devida à sua interação com tióis. As reações entre compostos de selênio e tióis foram estudadas com detalhes por TSEN \& TAPPEL (1958) e mais tarde por GANTHER (1968). Estes autores sugeriram que a toxicidade estava relacionada com a interação do selênio com tióis endógenos e a formação de selenotrissulfetos (RSSeSR) (Reação I), os quais podem ser reduzidos a selenopersulfetos (GSSeH) (Reação II) (Esquema 3) (GANTHER, 1971).

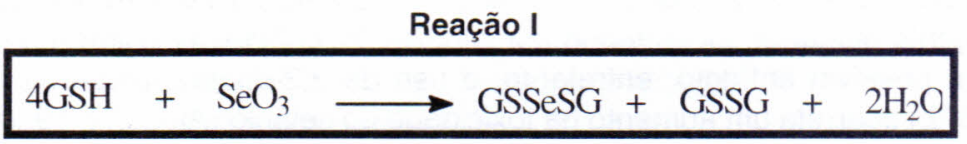

Reação II

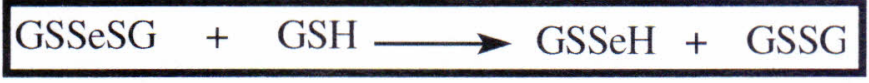

Esquema 3. Interação do selênio com tióis. GANTHER, 1971 
$\mathrm{Na}$ década de 60 , especulava-se sobre os efeitos tóxicos do selênio, uma vez que seu excesso causaria inativação de enzimas sulfidrílicas (TSEN \& COLLIER, 1959; SCHWARZ, 1961). Compostos orgânicos de selênio são capazes de inibir a atividade da enzima sulfidrílica delta-aminolevulinato dehidratase $(\delta-A L A-D)$ de fígado, rim e cérebro de ratos (BARBOSA et al., 1998; MACIEL, et al, 2000; JACQUES-SILVA et al., 2001; FARINA et al., 2001; BOLZAN, et al., 2002; PEROTTONI et al., 2003)

$\mathrm{Na}$ década de oitenta, SEKO e colaboradores (1989) propuseram a formação de superóxido $\left(\mathrm{O} 2^{\circ}\right)$, proveniente da reação de selenito com a glutationa. Alguns anos mais tarde, foi descrito que selenato, selenocisteína e selenometionina produzem superóxido na presença de glutationa, evidenciando assim os danos oxidativos como a provável causa da toxicidade do selênio (YAN \& SPALLHOZ, 1991).

As patologias mais comuns associadas à toxicidade do selênio são a doença alcalina (alkali disease), uma doença de caráter crônico, e o falso cambalear (blind staggers), uma manifestação clínica característica de comprometimento do SNC (MARTIN \& GERLACK, 1972).

De uma forma geral, as formas inorgânicas de selênio são mais tóxicas que as formas orgânicas. O selenito $\left(\mathrm{SeO}_{3}{ }^{2-}\right)$ é um oxidante biológico e é o mais tóxico entre as demais formas de selênio (GUPTA \& PORTER, 2001). A dose letal mínima para o selenito de sódio $\left(\mathrm{Na}_{2} \mathrm{SeO}_{3}=3,25-3,50 \mathrm{mg} \mathrm{Se} / \mathrm{kg}\right)$ é inferior a dose letal mínima para o arsenito de sódio $\left(\mathrm{Na}_{2} \mathrm{HAsO}_{3}=4,25-4,75 \mathrm{mg} \mathrm{As} / \mathrm{kg}\right.$ ) (para revisão ver FRANKE \& MOXON,1937).

Apesar de não existir um tratamento adequado para as intoxicações por selênio (selenoses), pode-se utilizar compostos que reduzam a sua toxicidade, tais como o ácido ascórbico (SVIRBELY, 1938), o arsênio (capaz de aumentar a excreção biliar), a metionina (administrada com vitamina $E$, as quais protegem o tecido hepático de necroses causadas por selenoses crônicas) (OKAMOTO \& GÜNTHER, 1972; WILBER, 1980). O tratamento com EDTA, um agente quelante, foi sugerido por SVIJAKOV \& BRAUN (1959) como um possivel antídoto; entretanto, o uso de 2,3-dimercaptopropanol (BAL) acarreta um aumento na toxicidade do selênio (AMDUR, 1958).

\section{Telúrio}

2.1. Histórico

O elemento telúrio foi descoberto em 1782; entretanto, a 
inclusão deste átomo em moléculas orgânicas ocorreu no início do século XIX. No Brasil, a utilização do telúrio em estudos químicos foi introduzida pelo Professor Reinbolt, o qual dedicou-se ao estudo sistemático de compostos orgânicos contendo telúrio e sua aplicabilidade como intermediários em síntese orgânica (PETRAGNANI, 1995; COMASSETO et al., 1997).

Os efeitos do telúrio sobre o organismo animal começaram a ser estudados com CHALLENGER (1955). Todavia, os primeiros relatos a respeito da toxicidade deste elemento aconteceram após o acidente de Windscale (UK), na Europa (STEWART \& CROOKS, 1958).

\subsection{Características químicas e atividades biológicas}

O telúrio pertence ao grupo 16 , da tabela periódica, assim como o enxofre e o selênio. Pode apresentar-se com diferentes números de oxidação, quais sejam: telurato $(\mathrm{Te}+6)$, telurito $(\mathrm{Te}+4)$, telúrio elementar (Te0) e telureto (Te-2) (SCANSETTI, 1992). Este elemento é encontrado com maior freqüência na forma de teluretos com o ouro, bismuto, chumbo e prata.

Da mesma forma que o selênio, o telúrio apresenta a propriedade de interagir com metais (PAINTER, 1941; KHAYAT \& DENCHER, 1984).

Os compostos organotelúrio apresentam propriedades antiinflamatórias e antivirais (SREDNI et al., 1987; SUN et al., 1996). Estudos recentes têm demonstrado que, tal como o Ebselen, os diteluretos de diarila, podem apresentar propriedade tiol peroxidase (ENGMAN et al., 1992; KANDA et al., 1999). Conseqüentemente, o emprego farmacológico destes agentes poderá crescer nos próximos anos, desde que não apresentem características tóxicas.

\subsection{Toxicologia}

Ao contrário do selênio, o telúrio não apresenta função fisiológica descrita até o momento (TAYLOR, 1996). Entretanto, as configurações eletrônicas do selênio e do telúrio são semelhantes; conseqüentemente, estes apresentam algumas características similares, assim como a toxicidade, que está relacionada ao estado de oxidação deste elemento (VAN VLEET et al., 1982).

Industrialmente, o telúrio é utilizado no manufaturamento de semicondutores e outros componentes eletrônicos (CLAYTON \& 
CLAYTON, 1981). Além disto, são empregados no processo de síntese de alguns fármacos e explosivos, na vulcanização de borracha, em lubrificantes sólidos e na petroquímica, entre outros (PAZ, 1989; TAYLOR, 1996).

Casos de intoxicação ocupacional aguda por telúrio são raros; entretanto, quando ocorrem, os sintomas são dores de cabeça, naúseas, alteração da freqüência cardíaca, bem como odor característico de alho (MÜLLER et al., 1989; TAYLOR, 1996).

O mecanismo proposto para explicar a toxicidade do telúrio, assim como para o selênio, envolve a oxi-redução de grupamentos SH de moléculas biologicamente ativas (BLAIS et al., 1972; YOUNG et al., 1981; DEUTICKE et al., 1992).

O telúrio na forma inorgânica é permeável à barreira placentária e, portanto teratogênico em ratos (AGNEW, 1972; LACASSE \& RICHTER, 1976). É um composto altamente tóxico, principalmente, para os mamíferos em desenvolvimento, podendo, inclusive, afetar a pele e outros orgãos, entre os quais, o rim (TAYLOR, 1996).

Da mesma maneira que o selênio, o telúrio na forma inorgânica apresenta-se mais tóxico que na forma orgânica. Além disso, telurito de sódio $\left(\mathrm{Na}_{2} \mathrm{TeO}_{3}=2,25-2,50 \mathrm{mg} \mathrm{Te} / \mathrm{kg}\right)$ possui dose letal mínima inferior à dose letal mínima do selenito $\left(\mathrm{Na}_{2} \mathrm{SeO}_{3}=3,25\right.$ - 3,50 mg Se/kg) e do arsenito de sódio $\left(\mathrm{Na}_{2} \mathrm{HAsO}_{3}=4,25-4,75 \mathrm{mg}\right.$ $\mathrm{As} / \mathrm{kg}$ ), demonstrando um efeito tóxico superior aos outros dois compostos (para revisão ver FRANKE \& MOXON, 1937).

Quando presentes na forma orgânica, os compostos de telúrio demonstram toxicidade superior aos compostos de selênio. Por outro lado, esta toxicidade difere entre as espécies animais e difere conforme a via de administração utilizada, intraperitoneal ou subcutânea (MEOTTI et al., 2003).

\section{Efeitos neurotóxicos induzidos por compostos de selênio e telúrio}

A toxicologia de compostos de selênio e telúrio tem sido alvo de interesse em virtude da descoberta de suas aplicações na síntese de compostos orgânicos e de suas propriedades biológicas (PETRAGNANI et al., 1976; COMASSETO, 1983; PARNHAM \& GRAF, 1991; KANDA et al., 1999). São importantes intermediários e 
reagentes muito utilizados em síntese orgânica (PAULMIER, 1986; BRAGA et al., 1996; 1997). Conseqüentemente, há risco de contaminação ocupacional por estes compostos.

Outro aspecto relevante é a tentativa crescente de desenvolvimento de compostos que possuam atividades biológicas e aplicações farmacológicas (PARNHAM \& GRAF, 1991; NOGUEIRA et al., 2003b; ROSSATO et al., 2002a). Sob este ponto de vista, este trabalho revisa a toxicidade de compostos de selênio e telúrio com ênfase nos efeitos sobre o sistema nervoso central (SNC).

Compostos orgânicos e inorgânicos de telúrio são altamente tóxicos ao SNC de roedores (D'GREGORIO \& MILLER, 1988). Causam hidrocefalia, hipomielinização ou desmielinização (D'GREGORIO \& MILLER, 1988; TOEWS et al., 1991; TAYLOR, 1996). Os efeitos da intoxicação pelo telúrio no SNC são demonstrados através da neuropatia periférica durante o período de mielinogênese ativa (LAMPERT \& GARETT, 1971; HARRY et al., 1989), afetando a transcrição das proteínas que formam a mielina (MORELL et al., 1994). A administração de telúrio em ratos provocou, além de alterações na mielina, danos em astrócitos situados no córtex cerebral (SMIALEK et al., 1994). Acredita-se que os efeitos do telúrio são expressos, principalmente, durante um período particular do desenvolvimento (WIDY-TYSZKIEWICZ, 2002).

A intoxicação pelo telúrio pode afetar o aprendizado e a memória em ratos, provavelmente, por afetar a integridade das áreas corticais e do hipocampo. A integridade hipocampal é crítica para as funções normais de memória e desta forma, é altamente vulnerável a danos neurotóxicos (WIDY-TYSZKIEWICZ, 2002).

O telúrio interfere na síntese de colesterol nas células de Schwann através da inibição da esqualeno monoxigenase (WAGNERREClO et al., 1991). Compostos de selênio também são hábeis em inibir a esqualeno monoxigenase, levando à interrupção da formação e degeneração da mielina (GUPTA \& PORTER, 2001). O efeito inibitório do telúrio e do selênio sobre a esqualeno monoxigenase, somado a ausência de inibição de outras enzimas implicadas na síntese de colesterol sugerem que o mecanismo inibitório seja devido à ligação dos compostos aos grupos sulfidrila de cisteínas vicinais da enzima (LADEN \& PORTER, 2001).

Estudos indicam que camundongos expostos ao ditelureto de difenila ( $\mathrm{PhTe})_{2}$ apresentam acentuada vacuolização de corpos celulares no cérebro. Por outro lado, a exposição de camundongos ao disseleneto de difenila ( $\mathrm{PhSe})_{2}$ não causa nenhuma alteração 
histológica que indique neurotoxicidade (NOGUEIRA et al., 2001a). Apesar da ausência de alterações histológicas em camundongos que receberam $(\mathrm{PhSe})_{2}$, houve uma clara inibição da enzima $\delta$-ALA-D de cérebro de camundongos expostos de forma aguda ou crônica ao $(\mathrm{PhSe})_{2}$ e ao (PhTe) $)_{2}$ (MACIEL et al., 2000).

A exposição a doses altas de disseleneto de difenila (250 $\mathrm{mmol} / \mathrm{kg}, \mathrm{s} . \mathrm{c}$ ) aumentou três vezes a quantidade de selênio total no cérebro de camundongos. Estes resultados sugerem que este órgão é um alvo potencial para a toxicidade dos organocalcogênios (JACQUES-SILVA et al., 2001).

Estudos recentes constataram que disselenetos orgânicos são capazes de induzir convulsão em camundongos. Neste trabalho observamos o envolvimento de mais de um sistema neuronal no episódio convulsivo, uma vez que moduladores alostéricos do sistema GABAérgico e um antagonista do sistema colinérgico foram capazes de diminuir ou anular o potencial convulsivo induzido pelo disseleneto de difenila (NOGUEIRA et al., 2003a).

O aminoácido L-glutamato é considerado o maior mediador de sinais excitatórios no SNC de mamíferos e está envolvido em muitos aspectos da função normal do SNC, incluindo cognição, memória e aprendizagem (FONNUM, 1984; OTTERSEN \& STORMMATHISEN, 1984; COLLINGRIDGE \& LESTER, 1989; HEADLEY \& GRILLNER, 1990). Simultaneamente, o glutamato também desempenha um papel importante no desenvolvimento do SNC, como a indução sináptica, migração, diferenciação e morte celular (MCDONALD \& JOHNSTON, 1990; KOMURO \& RAKIC, 1993; JOHNSTON, 1995; La MANTIA, 1995; VALLANO,1998). O glutamato exerce seu papel por ativação dos receptores glutamatérgicos. Estes receptores estão localizados nas membranas pré e pós-sinápticas, bem como nas membranas das células gliais (MELDRUM et al., 1999). Desta forma, a concentração de glutamato no fluído extracelular é que determina a "extensão" da estimulação dos receptores (DANBOLT, 2000).

O SNC contém alta concentração de glutamato, mas em condições fisiológicas, somente uma fração pequena deste glutamato apresenta-se nos fluídos extracelulares (HAMBERGER et al., 1983; LEHMANN et al., 1983). É fundamental que a concentração de glutamato extracelular seja baixa, uma vez que, a manutenção deste aminoácido na fenda sináptica poderá induzir uma excessiva ativação de seus receptores, causando excitotoxicidade (PRICE, 1999).

NOGUEIRA e colaboradores (2001b) observaram que o 
disseleneto e o ditelureto de difenila inibem a união de glutamato aos seus receptores, bem como a união de MK-801 ao subtipo de receptor glutamatérgico N-metil-D-aspartato (NMDA). Outros autores demonstraram que o organocalcogênio Ebselen é capaz de diminuir a funcionalidade do receptor NMDA por oxidar grupos $\mathrm{SH}$ deste receptor (HERIN et al., 2001). Estes dados corroboram com o efeito direto dos organocalcogênios sobre o sistema glutamatérgico.

Um efeito inibitório na união de glutamato aos seus receptores, bem como a união de MK-801 ao subtipo de receptor NMDA sugere que as propriedades toxicológicas dos organocalcogênios estejam relacionadas, em parte, a uma inibição dos sistemas de neurotransmissão excitatória. Evidências recentes têm mostrado que a manutenção da fisiologia do sistema glutamatérgico é essencial para o perfeito funcionamento do cérebro. De fato, um desequilíbrio pelo aumento ou pela diminuição da neurotransmissão glutamatérgica é altamente neurotóxico (OZAWA et al, 1998).

A união de nucleotídeos da guanina em proteínas $\mathrm{G}$ diminui a afinidade do glutamato pelos seus receptores. Os organocalcogênios foram hábeis em inibir a ligação de GMP-PNP em membranas sinápticas de ratos. Todavia, o efeito mais pronunciado foi na ligação a proteínas $\mathrm{G}$ (união estável), mostrando que estes compostos apresentam efeito extra e intracelular (NOGUEIRA et al., 2001b).

De fato, os organocalcogênios alteram a funcionalidade do sistema glutamatérgico. O disseleneto de difenila é capaz de inibir a captação de glutamato em vesículas, enquanto os compostos Ebselen e ditelureto de difenila inibem a captação de glutamato em vesículas e em sinaptossomas in vitro (NOGUEIRA et al., 2002). Em estudos in vivo, camundongos que apresentaram episódio convulsivo induzido por disseleneto de dipropila (C3H7Se) tiveram uma redução de $50 \%$ na captação de glutamato (NOGUEIRA et al., 2003a).

Os mecanismos da neurotoxicidade dos organocalcogênios são ainda pouco conhecidos. Uma hipótese provável envolve a oxidação de grupos tiólicos essenciais em proteínas alvo (MACIEL et al., 2000). A modulação de grupos tiólicos de transportadores e receptores de glutamato altera o funcionamento fisiológico do sistema glutamatérgico (DANBOLT, 2000).

Os subtipos de transportadores de glutamato EAAT1, EAAT2 e EAAT3 são regulados através do estado redox do resíduo de cisteína na estrutura do transportador (DANBOLT, 2000). Interconversões químicas reversíveis entre o estado reduzido e o oxi- 
dado são acompanhadas por mudanças dinâmicas na atividade de transporte. A atividade máxima é encontrada no estado reduzido, enquanto a mínima no estado oxidado do resíduo de cisteína (TROTTI et al., 1997).

Desta forma, substâncias redutoras ou oxidantes, de origem endógena ou exógena, são capazes de alterar o sistema de captação de aminoácidos excitatórios pelos transportadores. Os grupos sulfidrila do resíduo de cisteína são altamente sensiveis à oxidação (DANBOLT, 2000).

Da mesma forma, o complexo do receptor NMDA possui um sítio sensível à modulação redox (modulado tanto por oxidantes quanto por redutores) (GOZLAN \& BEM-ARI, 1995). A redução do sítio redox do receptor NMDA aumenta as respostas ao NMDA no SNC de mamíferos, enquanto agentes oxidantes podem diminuir suas respostas (HERIN et al., 2001).

Analisando os efeitos dos compostos de selênio e telúrio sobre o SNC, podemos concluir que o cérebro é um importante órgão alvo para a toxicologia destes compostos. Assim, há necessidade de mais pesquisas toxicológicas para o advento terapêutico destes compostos, devido, principalmente, a possíveis intoxicações ocupacionais. 


\section{Referências}

AGNEW, W.F. Transplacental uptake of 127 tellurium studied by wholebody autoradiography. Teratology 6: 331-338 (1972).

AMDUR, M.L. Tellurium oxide. An animal study in acute toxicity. Arch. Industr.Health 17: 665-667 (1958).

BARBOSA, N.B.V.; ROCHA, J.B.T.; ZENI, G.; EMANUELLI, T.; BEQUE, M.C.; BRAGA, A.L. Effect of inorganic forms of selenium on delta-aminolevulinate dehydratase from liver, kidney and brain of adult rats. Toxicol. Appl. Pharmacol. 149: 243-253 (1998).

BEDWALL, R.S.; NAIR, N.; SHARMA, M.P.; MATHUR, R.S. Selenium its biological perspectives. Med. Hypotheses 41: 150-159 (1993).

BEHNÉ, D. \& KYRIAKOPOULOS, A. Identification of type I iodothyronine 5'-deiodinase as a selenoenzyme. Biochem. Bioph. Res. Co. 173: 1143-1149 (1990).

BLAIS, F.X.; ONISCHUK, R.T.; DE MEIO, R.H. Hemolysis by tellurite In: The tellurite test for hemolysis. J. AOA. 73 (1972).

BOLZAN, R.C.; FOLMER, V.; FARINA, M.; ZENI, G.; NOGUEIRA, C.W.; ROCHA, J.B.T.; EMANUELLI, T. d-Aminolevulinate dehydratase inhibition by phenyl selenoacetylene. Effect of reaction with hydrogen peroxide. Pharmacol Toxicol. 90 (4): 214-9 (2002).

BRAGA, A.L.; SILVEIRA, C.C.; ZENI, G.; SEVERO, W.A.; STEFANI, H.A. Synthesis of selenocetais from enol ethers. J. Chem. Res. (S) 206-207 (1996).

BRAGA, A.L.; ZENI, G.; ANDRADE, L.H.; SILVEIRA, C.C. Stereoconservative formation and reativity of a-chalcogenfunctionalized vinylithium compounds from bromo-vinylic chalcogens. Synlett 5: 595-596 (1997).

CADENAS, E. \& SIES, H. Oxidative stress: excited oxygen species and enzyme activity. Adv. Enzyme Regul. 23: 217-237 (1985).

CLAYTON, G.D. \& CLAYTON, F.E. Patty's industrial hygiene and toxicology. Eds, John Wiley, Chilchester (1981).

COHEN, M.V. Free redicals in ischemic and reperfusion myocardial injury: is this time for clinical trials? Ann. Intern. Med. 111: 918931 (1989).

COLLINGRIDGE, G.L. \& LESTER, R.A.J. Excitatory amino acids receptors in the vertebrate central nervous system. Pharmacol. Rev. 40: 143-210 (1989).

COMASSETO, J.V. Vinylic selenides. J. Organomet. Chem. 253: 131-181 (1983). 
COMASSETO, J.V.; LING, L.W.; PETRAGNANI, N.; STEFANI, H.A. Vinylic selenides and tellurides - preparations, reactivity and synthetic applications. Synthesis-Stuttgart 4: 373 (1997).

CUVIN-ARALAR, M.L. \& FURNESS, R.W. Mercury and selenium interaction: a review. Ecotoxicol. Environ. Health 21: 348-364 (1991).

D'GREGORIO, R.E.P. \& MILLER, R.K. Teratogenicity of tellurium dioxide: prenatal assessment. Teratology 37: 307-316 (1988).

DAIBER, A.; ZOU, M.; BACHSCHMID, M.; ULLRICH, V. Ebselen as a peroxynitrite scavenger in vitro and ex vivo. Biochem. Pharmacol. 59: $153-160$ (2000)

DANBOLT, N.C. Glutamate uptake. Pro. Neurobiol. 65: 1-105 (2000). DEUTICKE, B.; LÜTKEMEIER, P.; POSE, B. Tellurite-induced damage of the erythrocyte membrane. Manifestations and mechanisms. Biochem. Biophys. Acta 1109: 97-107 (1992).

DIAZ, J.P.; NAVARRO, M.; LOPEZ, H.; LOPEZ, M.C. Determination of selenium levels in dairy products and drinks by hydride generation atomic absorption spectrometry: correlation with daily dietary intake. Food Addit. Contam. 14: 109-114 (1997).

ELLINGSEN, D.G.; HOLLAND, R.I.; THOMASSEN, Y.; LANDROOLSTAD, M.; FRENCH, W.; KJUUS, H. Mercury and selenium in workers previously exposed to mercury vapour at a chloroalkali plant. Brit. J. Ind. Med. 50: 745-752 (1993).

ENGMAN, L.; STERN, D.; COTGREAVE, I.A.; ANDERSSON, C.M. Thiol peroxidase activity of diaryl ditellurides as determined by a 1H NMR method. J. Am. Chem. Soc. 114: $9737-9743$ (1992).

EVANS, J.P.; WHITEMAN, M.; TREDGER, J.M.; HALLIWELL, B. Antioxidant properties of s-adenosyl-L-methionine: a proposed addition to organ storage fluids. Free Radical Bio. Med. 23: 10021008 (1997).

FARINA, M.; FOLMER, V.; BOLZAN, R.; ANDRADE, L.H.; ZENI, G. Selenoxides inhibit d-aminolevulinic acid dehydratase. Toxicol. Lett. 119: 27-37 (2001).

FAVIER, A.E. In: Schulemmer U. Nutritional and clinical factors affecting the bioavailability of trace elements in humans. Germany: Ettlingen, 1993. 93, 202-211 Bioavailability.

FLOYD, R.A. Role of oxigen free radicals in carcinogenesis and brain ischemia. FASEB J. 4: 2587-2597 (1990).

FONNUM, F. Glutamate: a neurotransmitter in mammaliam brain. J. Neurochem. 42: 1-11 (1984). 
FRANKE, K.W. A new toxicant occurring naturally in certain samples of plants foodstuffs. I. Results obtained in preliminary feeding trials. J. Nutr. 8: 597-608 (1934).

FRANKE, K.W. \& MOXON, A.L. The toxicity of orally ingested arsenic , selenium, tellurium, vanadium and molybdenum. J. Pharmacol. 61: 89-102 (1937).

FROST, D.V. \& LISH, P.M. Selenium in biology. Annu. Rev. 15: 259284 (1983).

GANTHER, H.E. Selenotrisulfides. Formation by reaction of thiols with selenious acid. Biochemistry 7: 2898-2905 (1968).

GANTHER, H.E. Reduction of the selenotrisulfide derivative of glutathione to a persulfide analog by glutathione reductase. Biochemistry 10: $4089-4098$ (1971).

CHALLENGER, F. Biological methylation. Q. Chem. Rev. Soc. 9: 255-286 (1955).

GOZLAN, H. \& BEM-ARI, Y. NMDA receptor redox sites: are they targets for selective neuronal protection? TiPS 16: 368-375 (1995).

GUPTA, N. \& PORTER, T.D. Inhibition of human squalene monooxygenase by selenium compounds. J. Biochem. Mol. Toxicol. 16: 18-23 (2001).

HALLIWELL, B. Antioxidant characterization: methodology and mechanism. Biochem. Pharm. 49: 1341-1348 (1995).

HALLIWELL, B. Vitamin C: poison, prophylactic or panacea? Trends Biochem. Sci. 24: 255-257 (1999).

HALLIWELL, B. \& GUTTERIDGE, J.M.C. Role of free radicals and catalytic metal ions in human disease: an overview. Method. Enzymol. 186: 1-5 (1990).

HAMBERGER, A.; BERTHOLD, C.; KARLSSON, B.; LEHMANN, A.; NYSTROM, B. Extracellular GABA, glutamatte and glutamine in vivo - perfusion-dialysis of rabbit hippocampus. Neurol. Neurobiol. 7: 473-492 (1983).

HARRY, G.J.; GOODRUM, J.F.; BOULDIN, T.W.; WAGNER-RECIO, M.; TOEWS, A.D.; MORELL, P. Tellurium-induced neuropathy: metabolic alterations associates with demyelination and remyelination in rat sciatic nerve. J. Neurochem. 52: 938-945 (1989).

HEADLEY, P.M. \& GRILLNER, S. Excitatory amino acids and synaptic transmission: the evidence for a physiological function. Trends Pharmacol. Sci. 11: 205-211 (1990).

HERIN, G.A., DU, S., AIZENMAN, E. The neuroprotective agent Ebselen modifies NMDA receptor function via the redox modulatory 
site. J. Neurochem. 78: 1037-1314 (2001).

IMAI, H.; GRAHAM. D.I.; MASAYASU, H.; MACRAE, I.M. Antioxidant Ebselen reduces oxidative damage in focal cerebral ischemia. Free Rad. Biol. Med. 34, 56-63 (2003).

JACQUES-SILVA, M.C.; NOGUEIRA, C.W.; BROCH, L.C.; FLORES, E.M.M. Diphenyl diselenide and ascorbic acid changes deposition of selenium and ascorbic acid in liver and brain of mice. Pharmacol. Toxicol. 88: 119-125 (2001).

JANSSEN, A.M.; BOSMAN, C.B.; SIER, C.F.; GRIFFIOEN, G. SODS in relation to the overall survival of colorectal cancer patients. Brit. J. Cancer 78: 1051-1057 (1998).

JOHNSTON, M.V. Neurotransmitters and vulnerability of the developing brain. Brain Dev. 17: 301-306 (1995).

JOURD`HEUIL, D.; MILLS, L.; MILES, A.M.; GRISHAM, M.B. Effect of nitric oxide on hematoprotein-catalyzed oxidative reactions. Nitric Oxide 2: 37-42 (1998).

KANDA, T.; ENGMAN, L.; COTGREAVE, I.A.; POWIS, G. Novel watersoluble diorganyl tellurides with thiol peroxidase and antioxidant activity. J. Org. Chem. 64: 8161-8169 (1999).

KHAYAT, A. \& DENCHER, L. Interactions between tellurium and mercury in murine lung and other organs after metallic mercury inhalation: a comparasion with selenium. Chem. Biol. Interac. 50: 123-133 (1984).

KLAYMAN, D.L. \& GÜNTHER, W.H. (Eds), Organic selenium compounds: their chemistry and biology, John Wiley and sons, New York, 68-157pp (1973).

KOMURO, H. \& RAKIC, P. Modulation of neuronal migration by NMDA receptors. Science 260: 95-97 (1993).

KONDOH, S.; NEGASAWA, S.; KAWANISHI, M.; YAMAGUSHI, K.; KAJIMOTO, S.; OHTA, T. Effects of ebselen on cerebral ischemis and reperfusion evaluated by microdialysis. Neurol. Res. 21: 682686 (1999).

KRISHNA, M.C.; RUSSO, A.; MITCHELL, J.B.; GOLDSTEIN, S. Do nitroxide antioxidants act as scavengers of superoxide or as superoxide dismutase mimics? J. Biol. Chem. 271: 26026-26031 (1996).

La MANTIA, A.S. The usual suspects: GABA and glutamate may regulate proliferation in the neocortex. Neuron 15: 1223-1225 (1995).

LACASSE, Y. \& RICHTER, C. Toxicité du sélénium et de ses dérivés. Union Med. Can. 1192-1199 (1976). 
LADEN, B. \& PORTER, T. Inhibition of human squalene monooxygenase by tellurium compounds. Evidence of interaction with vicinal sulfhydryls. J. Lipid Res. 42: 235-240 (2001).

LAMPERT, P.W. \& GARETT, R.S. Mechanism of demyelination in tellurium neuropathy. Electron microscopic observation. Lab. Invest. 25: 380-388 (1971).

LEHMANN, A.; ISACSSON, H.; HAMBERGER, A. Effects of in vivo administration of kainic acid on the extracellular amino acid pool in the rabbit hippocampus. J. Neurochem. 40: 1314-1320 (1983).

LEVANDER, O.A. \& BURK, R.F.. In: Shils M.E., Olson J.Á, Shike , M. Modern nutrition in health and disease. Philadelphia: Lea and Febiger, 1994, 242-251, Selenium.

LEVANDER, O.A.; MORRIS, O.A.; HIGGS, D.J. Selenium as a catalyst for the reduction of cytochrome c by glutathione. Biochemistry 6: $4591-4595$ (1983).

$\mathrm{LI}, \mathrm{Y} \& \mathrm{CAO}, \mathrm{Z}$. The neuroprotectant ebselen inhibits oxidative DNA damage induced by dopamine in the presence of copper ions. Neurosci. Lett. 330: 69-73 (2002).

LITOV, R.E. \& COMBS, G.F. El selenio en nutrición pediátrica. Pediatrics 31: 159-171 (1991).

MACIEL, E.N.; BOLZAN, R.C.; BRAGA, A.L.; ROCHA, J.B.T. Diphenyl diselenide and diphenyl ditelluride differentialy affect $d$ Aminolevulinate dehydratase from liver, kidney and brain of mice. J. Biochem. Mol. Toxicol. 14: 310-319 (2000).

MARTIN, J.L. \& GERLACK, M.L. Selenium metabolism in animals. Ann. NY Acad. Sci. 192: 193-199 (1972).

MATÉS, J.M. Effects of antioxidant enzymes in the molecular control of reactive oxygen species toxicology. Toxicology 153: 83-104 (2000).

McDONALD, J.W. \& JOHNSTON, M.V. Physiological and pathophysiological roles of excitatory amino acids during central nervous system development. Brain Res. Rev. 15: 41-70 (1990).

McKENZIE, R.C.; RAFFERTY, T.S.; BECKETT, G.J. Selenium: an essential element for immune function. Immunol. Today 19: 324345 (1998).

MELDRUM, B.S.; AKBAR, M.T.; CHAPMAN, A.G. Glutamate receptors and transporters in genetic and acquired models of epilepsy. Epilepsy Res. 36: 189-204 (1999).

MELOV, S.; SCHNEIDER, J.A.; DAY, B.J.; HINERFELD, D.; COSKUN, P. A novel neurological phenotype in mice lacking 
mitochondrial manganese superoxide dismutase. Nat. Genet. 18: 159-163 (1998).

MEOTTI, F.C.; BORGES, V.C.; ZENI, G.; ROCHA, J.B.T.; NOGUEIRA, C.W. Potential Renal and Hepatic Toxicity of Diphenyl Diselenide, Diphenyl Ditelluride and Ebselen for Rats and Mice. Toxicol. Lett. 143: 9-16 (2003).

MORELL, P.; TOEWS, A.D.; WAGNER, M.; GOODRUM, J.F. Gene expression during tellurium-induced primary demyelination. Neurotoxicology 15:171-180 (1994).

MOUSSAOUI, S.; OBINU, M.C.; DANIEL,N.; REIBAUD, M.; BLANCHARD, V.; IMPERATO, A. The antioxidant Ebselen prevents neurotoxicity and clinical symptoms in a primate model of Parkinson's disease. Exp. Neurol. 166: 235-245 (2000).

MÜLLER, A.; CADENAS, E.; GRAF, P.; SIES, H. A novel biologically active seleno-organic compound. In: Glutathione peroxidase-like activity in-vitro and antioxidant capacity of PZ-51 (ebselen).

Biochem. Pharmacol. 33: 3235-3239 (1984).

MÜLLER, R.; ZSCHIESCHE, W.; STEFFEN, H.M.; SCHALLER, K.H.

Tellurium intoxication. Klin. Wocherschr. 67: 1152-1155 (1989).

NAVARRO-ALARCÓN, M. \& LÓPEZ-MARTINEZ, M.C. Essentiality of selenium in the human body: relationship with different diseases. Sci. Total Environ. 249: 347-371 (2000).

NEVE, J.; HENRY, M.; PERETZ, A. \& MARESCHI, J.P. L'importance nutritionnelle du sélènium. Cah. Nutr. Diet 22: 145-162 (1987).

NOGUEIRA, C.W., MACIEL, E.N., ZENI, G., GRAÇA, D., ROCHA, J.B.T. Biochemical toxicology of simple diorganyl chalcogenides. ECSOC, http://www.mdpi.net/ecsoc-5/, [d0013] (2001a).

NOGUEIRA, C.W; MEOTTI, F.C.; CURTE, E.; PILISSÃO, C.; ZENI, G.; ROCHA, J.B.T. Investigations into potential neurotoxicity induced by diselenides in mice and rats. Toxicology 183: 29-37 (2003a).

NOGUEIRA, C.W.; QUINHONES, E.B.; JUNG, E.A.C.; ZENI, G.; ROCHA, J.B.T. Anti-inflammatory and antinociceptive activity of diphenyl diselenide. Inflamm. Res. 52: 56-63 (2003b)

NOGUEIRA, C.W.; ROTTA, L.N.; PERRY, M.L.; SOUZA, D.O.; ROCHA, J.B.T. Diphenyl diselenide and diphenyl ditelluride affect the rat glutamatergic system "in vitro" and "in vivo". Brain Res. 906: 157-163 (2001b).

NOGUEIRA, C.W.; ROTTA, L.N.; ZENI,G.; SOUZA, D.O.; ROCHA, J.B.T. Exposure to Ebselen changes glutamate uptake and release 
by rat brain synaptosomes. Neurochem. Res. 3: 283-288 (2002).

OKAMOTO, Y. \& GÜNTHER, W.H. Organic selenium and tellurium chemistry. Ann.NY Acad. Sci Part lii. 1972. Bilogical aspects of organic selenium and tellurium compounds - Metabolic interrelationship and adaptations in selenium toxicity.

ORTUNÕ, J.; ROS, G.; PERIAGO, M.; MARTINEZ, C.; LOPEZ, G. Selenium bioavailability and methods of evaluation. Food Sci. Technol. Int. 2: 135-150 (1996).

OTTERSEN, O.P. \& STORM-MATHISEN, J. In: Björklund, A., Hökfelt, T., Kuhar, M.J. Neurons containing or accumulating transmitter amino acids. Handbook of Chemical Neuroanatomy. Amsterdam: Elsevier, 1984. Part II 3: 141-246. Classical Transmitters and Transmitter Receptors in the CNS.

OZAWA, S.; KAMIYA, H.; TSUZUKI, K. Glutamate receptors in the mammalian central nervous system. Prog. Neurobiol. 54: 581-618 (1998).

PAINTER, E.P. The chemistry and toxicity of selenium compounds, with special reference to the selenium problem. Chem. Rev. 28: 179-213 (1941).

PARKES, T.L.; ELIA, A.J. DICKINSON, D.; HILLIKER, A.J. Extension of Drosophila lifespan by overexpression of human SOD1 in motorneurons. Nat. Genet. 19: 171-174 (1998).

PARNHAM, M.J. \& GRAF, E. Pharmacology of synthetic organic selenium compounds. Prog. Drug Res. 36: 10-47 (1991).

PAULMIER, C. Organic Synthesis Selenium. Oxford: Pergamon, 1986. Reagents and Intermediates.

PAZ, M.J.D. Espécies acetilênicas vinílicas e alquílicas contendo telúrio. Tese de doutorado. São Paulo, Departamento de Química, Universidade de São Paulo, USP (1989).

PEROTTONI, J.; RODRIGUES, O.E.D.; PAIXÃO, M.W.; ZENI, G.; LOBATO, L.P.; BRAGA, A.L.; ROCHA, J.B.T.; EMANUELLI, T. Renal and hepatic ALA-D activity and selected oxidative stress parameters of rats exposed to inorganic mercury and organoselenium compounds. Food. Chem. Toxicol. In press (2003).

PETRAGNANI, N. In: Mckillop A. Comprehensive organometallic chemistry II. Exeter, UK, Pergamon Press, 1995.

PETRAGNANI, N.; RODRIGUES, R.; COMASSETO, J.V. Reaction of selenenyl halides with wittig reagents. J. Organomet. Chem. 114: 281-292 (1976).

PORCIIUNCULA, L.O.; ROCHA, J.B.T.; BOECK. C.R.; VENDITE, D.; 
SOUZA, D.O. Ebselen prevents excitotocity provoked by glutamate in rat cerebellar granule neurons. Neurosci. Lett. 299: 271-220 (2001).

PRATICÒ, D. \& DELANTY, N. Oxidative injury in diseases of the central nervous system: focus on Alzheimer's disease. Am. J. Med. 109: 577-585 (2000).

PRICE, D.L. New order from neurological disorders. Nature 399: A3A5 (1999).

ROSSATO, J.I.; KETZER, L.A.; CENTURIÃO, F.B.; SILVA, S.J.N.; LÜDTKE, D.S.; ZENI, G.; BRAGA, A.L.; RUBIN, M.A.; ROCHA, J.B.T. Antioxidant properties of new chalcogenides against lipid peroxidation in rat brain. Neurochem.I Res. 3: 297-303 (2002a).

ROSSATO, J.I.; ZENI, G.; MELLO, C.F.; RUBIN, M.A.; ROCHA, J.B.T. Ebselen blocks the quinolinic acid-induced production of thiobarbituric acid reactive species but does not prevent the behavioral alterations produced by intra-striatal quinolinic acid administration in rat. Neurosci. Lett. 318: 137-140 (2002b).

ROTRUCK, J.T.; POPE, A.L.; GANTHER, H.E.; SWANSON, A.B.; HAFEMAN, D.G.; HOEKSTRA, W.G. Selenium: biochemical role as a component of glutathione peroxidase. Science 179: 588-590 (1981).

SAITO, Y.; HASHIMOTO, T.; SASAKI, M.; HANAOKA, S.; SUGAI, $K$. Effect of selenium deficiency on cardiac of individuals with severe disabilities under long- term tube feeding. Dev. Med. Child. Neurol. 40: 743-748 (1998).

SCANSETTi, G. Exposure to metals that have recently come into use. Sci. Total Environ. 120: 85-91 (1992).

SCHWARTZ, K. \& FOLTZ, C.M. Selenium as a integral part of factor 3 against dietary necrotic liver degeneration. J. Am. Chem. Soc. 79: 200-214 (1957).

SCHWARZ, K. A possible site of action for vitamin E in intermediary metabolism. Amer. J. Clin. Nutr. 9: 71 (1961).

SEKO, Y.; SAITO, Y.; KITAHARA, J.; IMURA, N. Selenium in biology and medicine. Berlin: Springer-Verlag., 1989, 70-73 Active oxygen generation by the reaction of selenite with reduced glutathione in vitro.

SIES, H. Ebselen, a selenoorganic compound as glutathione peroxidase mimic. Free Rad. Biol. Med. 14: 313-323 (1993).

SMIALEKM.; GAJKOWSKA, B.; OTREBSKA, D. Electron Microscopy studies on the neurotoxic effect of sodium tellurite in 
the central nervous system of the adult rat. J. Brain Res. 35: 223232 (1994).

SMITH, A.M. \& PICCIANO, M.F. Relative bioavailability of seleniumcompounds in the lactating rat. J. Nutr. 117: 725-731 (1987).

SPALLHOLZ, J.E. On the nature of selenium toxicity and carcinostatic activity. Free Rad. Biol. Med. 20: 131-145 (1993).

SREDNI, B.; CASPI, R.R.; KALECHMAN, Y.; DANZIGER, Y.; BENYA'AKOV TAMARI, T. SHALIT, F.; ALBECK, M. A new immunomodulating compound (AS-101) with potential therapeutic application. Nature 330: 173-176 (1987).

STADTMAN, T.C. Selenium-dependent enzymes. Annu. Rev. Biochem. 49: 93-110 (1980).

STEWART, N.G. \& CROOKS, R.N. Long-range travel of the radioactive cloud from the accident at Windscale. Nature 182: 627-628 (1958).

SUN, X.; WONG, J.R.; SONG, K.; CHEN, L.B. Anticarcinoma activity of a novel drug, 3-ethyl-3'methyl-thiatelluracarbocyanine iodite (Te) a tellurium- containing cyanine targeted at mitochondria. Clin. Canc. Res. 2: 1335-1340 (1996).

SVIJAKOV, K.I. \& BRAUN, N.A. A possible site of action for vitamin E in intermediary metabolism. Am. J. Clin. Nut. 9: 71 (1959).

SVIRBELY, J.L. Vitamin C studies in the rat. The effect of selenium dioxide, sodium selenate and tellurate. Biochem. J. 32: 467 (1938).

TAKASAGO, T.; PETERS, E.E.; GRAHAM, D.I.; MASAYASU, H., MACRAE, I.M. Neuroprotective efficacy of ebselen, an antioxidant with anti-inflammatory actions, in a rodent model of permanent middle cerebral artery occlusion. Br. J. Pharmacol. 122: 12511256 (1997).

TAN, Y.X.; LI, W.H.; TAO, X.B.; JIANG, Y.Y.; CHEN, W.P.; ZHOU, B. Protection of ebselen against anoxic damage of culture neurons of cerebral cortex. Acta Pharmacol. Sin. 18: 201-203 (1997).

TAYLOR, A. Biochemistry of tellurium. Biol. Trace Elem. Res. 55: 231-239 (1996).

TOEWS, A.D.; ECKERMANN, C.E.; ROBERSON, M.D.; LEE, S.Y.; MORELL, P. Primary demyelination induced by exposure to tellurium alters mRNA levels for nerve growth factor receptor, SCIP, 2'3'-cyclic nucleotide 3'-phosphodiesterase, and myelin proteolipid protein in rat sciatic nerve. Mol. Brain Res. 11: $321-325$ (1991).

TRELEASE, S.F. \& BEATH, O.A. Selenium: its geological occurrence and its biological effects in relation to botany, chemistry, agriculture, nutrition and medicine. Burlington, VT: The Champlain Printers (1949). 
TROTTI, D.; NUSSBERGER, S.; VOLTERRA, A.; HEDIGER, M.A. Differential modulation of the uptake currents by redox interconversion of cysteine residues in the human neuronal glutamate transporter EAAC1. Eur. J. Neurosci. 9: 2207-2212 (1997).

TSEN, C.C. \& COLLIER, H.B. Selenite as a relatively weak inhibitor of some sulfhydryl enzime systems. Nature 183: 1327 (1959).

TSEN, C.C. \& TAPPEL, A.L. Catalytic oxidation of glutathione and other sulphydril compounds biselenite. J. Biol. Chem. 233: 12301232 (1958).

URSINI, F. \& BINDOLI, A. The role of selenium peroxidases in the protection against oxidative damage of membranes. Chem. Phys. Lipids 44: 255-276 (1987).

URSINI, F.; MAIORINO, M.; VALENTE, M.; FERRI, K.; GREGOLIN, C. Purification of pig liver of a protein which protects lipossomes and biomembranes from peroxidative degradation and exhibits glutathione peroxidase activity on phosphatidylcholine hydroperoxidase. Biochem. Biophys. Acta 710: 197-211 (1982).

VALLANO, M.L. Developmental aspects of NMDA receptor function. Crit. Rev. Neurobiol. 12: 177-204 (1998).

VAN VLEET, J.F.V. \& FERRANS, V.J. Ultrastructural alterations in skeletal muscle of ducklings fed selenium-vitamin E-deficient diet. Am. J. Vet. Res. 38: 1399-1405 (1982).

WAGNER-RECIO, M.; TOEWS A.D.; MORELL, P. Tellurium blocks cholesterol synthesis by inhibiting squalene metabolism: Preferential vulnerability to this metabolic block leads to peripheral nervous system demyelination. J. Neurochem. 57: 1981-1901 (1991).

WENDEL, A.; FAUSEL, M.; SAFAYHI, H.; TIEGS, G. A novel biologically active seleno-organic compound - II. Activity of PZ 51 in relation to glutathione peroxidase. Biochem. Pharmacol. 33: 3241-3245 (1984).

WHANGER, P.D.; PEDERSEN, N.D.; HATFIELD, J.; WESWING, P.H. Absortion of selenite and selenomethionine from ligated digestive tract segments in rats. Proc. Soc. Exp. Biol. Med. 153: 295-297 (1976).

WIDY-TYSZKIEWICZ, E.; PIECHAL, A.; GAJKOWSKA, B.;SMIALEK, M. Tellurium-induced cognitive deficits in rats are related to neuropathological changes in the central nervous system. Toxicol. Lett. 131: 203-214 (2002). 
WILBER, C.G. Toxicology of selenium: a review. Clin. Toxicol. 17(2): $171-230$ (1980).

WILLMORE, L.J. Post-traumatic epilepsy: cellular mechanisms and implications for treatment. Epilepsia 31: 67-73 (1990).

WILSON, S.R.; ZUCKER, P.A.; HUANG, R.R.C.; SPECTOR, A. Development of synthetic compounds whit glutathione peroxidase activity. J. Am. Chem. Soc. 111: 5936-5939 (1989).

YAMAGUCHI, T.; SANO, K.; TAKAKURA, K.; SAITO, I.; SHINOHARA, Y.; ASANO, T.; YASUHARA, $\mathrm{H}$. Ebselen in acute ischemic stroke. A placebo-controlled, double-blind clinical trial. Stroke 29: 12-17 (1998).

YAN, L. \& SPALLHOLZ, J.E. Free radical generation by selenium compounds. FASEB J 5A: 581 (1991).

YOUNG, V.R.; NAHAPETIAU, A.; JONGHORBONI, M. Selenium bioavailability with reference to human nutrition. Am. J. Clin. Nut. 35: 1076-1088 (1981).

Flavia Carla Meotti

Cristina Wayne Nogueira

Departamento de Química - CCNE Universidade Federal de Santa Maria

Santa Maria, RS - Brasil 
188 Ciência \& Natura, Santa Maria, 25: 163 - 187, 2003 